\title{
Nomofobia entre discentes de medicina e sua associação com depressão, ansiedade, estresse e rendimento acadêmico
}

\author{
Nomophobia among medical students and its association with depression, anxiety, stress and academic performance \\ Marcos Kubrusly' (10|mmkubrusly@gmail.com \\ Paulo Goberlânio de Barros Silva' (1) paulo_goberlanio@yahoo.com.br \\ Gabriel Vidal de Vasconcelos' (1) gabrielvidal17@gmail.com \\ Emanuel Delano Lima Gonçalves Leite' 1 (1) emanueldelano.ed@gmail.com \\ Priscilla de Almeida Santos' ${ }^{1}$ (1) pri_santos@hotmail.com \\ Hermano Alexandre Lima Rocha² (1) hermano@ufc.br
}

\begin{abstract}
RESUMO
Introdução: À medida que o mundo se torna cada vez mais interconectado, a adoção de tecnologia continua sendo um dos fatores definidores do progresso humano. A nomofobia representa uma condição mental causada pelo medo de ficar sem celular. Tal condição está diretamente associada à depressão, à ansiedade e ao estresse. Ainda, a nomofobia pode levar a danos cerebrais estruturais.

Objetivo: O presente estudo visa conhecer o efeito da nomofobia nos estudantes de Medicina de uma faculdade privada e sua associação com depressão, ansiedade, estresse e rendimento acadêmico.

Método: Trata-se de um estudo observacional de corte transversal, do qual participaram estudantes do curso de Medicina do Centro Universitário Christus. O distúrbio foi mensurado por meio do Questionário sobre Nomofobia (NMP-Q). Esse instrumento tem 20 questões, todas baseadas na escala do tipo Likert de 7 pontos, a qual foi validada para o português brasileiro. A depressão, a ansiedade e o estresse foram mensurados pelo instrumento DASS-21, uma versão simplificada da DASS. Validou-se também o questionário DASS-21 para o português brasileiro. 0 rendimento acadêmico foi mensurado por meio do IRA, fruto de uma complexa operação matemática que resulta em uma nota média do aluno no semestre e funciona como um índice de referência para o acompanhamento pedagógico na faculdade estudada. Além disso, estudaram-se os hábitos de uso do dispositivo. Apresentaram-se os resultados descritivos, e realizaram-se análises bivariadas de associação e correlação. Esse estudo foi aprovado pelo Comitê de Ética em Pesquisa.

Resultado: Obteve-se uma amostra de 292 estudantes. Praticamente todos os alunos (99,7\%) apresentaram algum grau de nomofobia, e $64,5 \%$ demonstraram nível moderado ou grave de nomofobia. Mais de $50 \%$ dos estudantes apresentaram graus superiores ao nível leve de estresse, e $19,5 \%$ e $11,2 \%$ dos estudantes manifestaram ansiedade e depressão graves ou muito graves, respectivamente. Quando se analisou a correlação dos escores no NMP-Q com os escores da DASS-21, observou-se que aumentos nessa pontuação levam à elevação do escore geral da DASS ( $p$ < 0,001 ) e que piores resultados na DASS-21 estão associados ao pior IRA.
\end{abstract}

Conclusão: Nosso estudo sugere que a nomofobia pode provavelmente aumentar a ansiedade, o estresse e a depressão, e, como consequência, levar uma baixa do rendimento acadêmico.

Palavras-chave: Smartphone; Angústia Psicológica; Avaliação Educacional.

\begin{abstract}
Introduction: As the world becomes increasingly interconnected, the adoption of technology remains one of the defining factors of human progress. Nomophobia (NO MObile PHOne PhoBIA) represents a mental condition caused by the fear of being detached from mobile phone connectivity. Such condition is directly associated with depression, anxiety, and stress. Moreover, nomophobia can lead to structural brain damage.

Objective: The present study aims to assess the effect of nomophobia on medical students at a private institution and its association with depression, anxiety, stress and academic performance.

Method: This is a cross-sectional observational study carried out in medical students at Centro Universitário Christus. Nomophobia was measured using the Nomophobia Questionnaire (NMP-Q). The NMP-Q has 20 questions, which are asked on a 7-point Likert scale. This scale has been validated for the Brazilian Portuguese language. Depression, anxiety and stress were measured by the DASS-21, a simplified version of the DASS instrument. The DASS-21 questionnaire was also validated for the Brazilian Portuguese language. Academic performance was measured through API, the product of a complex mathematical operation that results in the student's average grade in the semester and functions as a reference index for pedagogical follow-up in the assessed institution. In addition, the device use habits were assessed. Descriptive results were presented, and bivariate analyses of association and correlation were performed. This study was approved by the research ethics committee.
\end{abstract}

Result: A sample of 292 students was assessed. Virtually all students (99.7\%) had some degree of nomophobia, and 64.5\% had a moderate or severe level of nomophobia. More than $50 \%$ of the students had higher than mild degrees of stress, and $19.5 \%$ and $11.2 \%$ of the students had severe or very severe levels of anxiety and depression, respectively. When analyzing the correlation of NMP-Q with DASS-21 scores, it was observed that increases in NMP- $Q$ lead to increases in the overall DASS score $(p<0.001)$, and that worse results in DASS-21 are associated with worse API.

Conclusion: Our study suggests that nomophobia is likely to increase anxiety, stress and depression and, as a result, leads to a decrease in academic performance. Keywords: Smartphone; Psychological Distress; Educational Measurement.

${ }^{1}$ Centro Universitário Christus, Fortaleza, Ceará, Brasil.

2 Universidade Federal do Ceará, Fortaleza, Ceará, Brasil.

Editora-chefe: Rosiane Viana Zuza Diniz. | Editora associada: Rosana Alves.

Recebido em 22/10/20; Aceito em 19/06/21. | Avaliado pelo processo de double blind review. 


\section{INTRODUÇÃO}

À medida que o mundo se torna cada vez mais interconectado, tanto econômica quanto socialmente, a adoção de tecnologia continua sendo um dos fatores definidores do progresso humano'. De modo especial, o smartphone é o principal meio para o uso da tecnologia disponível na atualidade, cujo uso é muito comum na contemporaneidade. Por exemplo, no Reino Unido, $93 \%$ da população tem um smartphone ${ }^{2}$. Os smartphones têm a oferecer muitas recompensas, como melhora das habilidades sociais, entretenimento e preservação da identidade social ${ }^{3}$.

Porém, trata-se de um hábito que pode tornar-se uma adicção, sendo um problema comum entre adultos no mundo inteiro ${ }^{4}$, e, a partir disso, poderão ocorrer muitos eventos danosos nos âmbitos social, mental e acadêmico ${ }^{5}$. Em consequência desse uso inadequado, surge a nomofobia. O termo nomofobia vem da expressão inglesa no mobile phone phobia. Trata-se de uma condição mental causada pelo desconforto decorrente da impossibilidade de comunicação por meios virtuais ${ }^{6}$; no caso abordado neste estudo, o indivíduo sente medo quando não está com um dispositivo eletrônico, mais comumente o smartphone. Ademais, a nomofobia está diretamente associada à depressão, à ansiedade e ao estresse ${ }^{7,8}$. Ainda sob essa ótica, a Organização Mundial da Saúde (OMS) considera a adicção como doença, e, por conta disso, é imprescindível que haja mais investigação sobre esse problema que é comum na sociedade. Outrossim, faz-se necessário compreender que a adicção por smartphones está intimamente relacionada com a nomofobia, podendo, inclusive, ter as mesmas características ${ }^{9}$.

Em uma pesquisa, demonstrou-se que 60\% dos estudantes de Medicina da amostra tinham nomofobia moderada; e 22,1\%, grave ${ }^{10}$. Em outro estudo, constatou-se uma prevalência ainda maior entre acadêmicos de Medicina: $100 \%$ apresentavam essa condição ${ }^{11}$. Tal problema pode levar a vários efeitos adversos, como a diminuição da conectividade entre o córtex orbitofrontal e o núcleo accumbens. Além disso, foram observados sintomas de retirada e maior concentração de cortisol sérico ${ }^{12}$. Do ponto de vista estrutural, em um estudo europeu, observou-se que indivíduos com adicção ao smartphone apresentavam uma menor substância cinzenta na região inferior temporal, no córtex para-hipocampal e na região insular anterior esquerda ${ }^{13}$.

A nomofobia é uma patologia bastante prevalente entre os estudantes, sobretudo em acadêmicos da área da saúde. Embora o conhecimento e o domínio desse assunto sejam importantes, poucos estudos têm sido realizados em estudantes da área da saúde no Brasil; portanto, necessita-se de mais esclarecimentos para entendimento do tema. Essa escassez de informação é o que embasa a relevância da pesquisa aqui apresentada, a qual visa, portanto, conhecer a prevalência da nomofobia nos estudantes de Medicina e sua associação com depressão, ansiedade, estresse e rendimento acadêmico.

\section{MÉTODO}

\section{Caracterização do estudo e amostra}

Trata-se de um estudo observacional de corte transversal, do qual participaram estudantes do curso de Medicina do Centro Universitário Christus (Unichristus). Para o cálculo amostral, considerou-se a prevalência de nomofobia recentemente identificada entre estudantes de Odontologia, em estudo transversal, de 24,12\%, um erro tipo II de $20 \%$ (poder de $80 \%$ ) e um nível de significância de $95 \%$, chegando-se ao n amostral mínimo de 305 indivíduos ${ }^{14}$. Adotaram-se os seguintes critérios de inclusão: acadêmicos adequadamente matriculados, presença de, pelo menos, $75 \%$ nas aulas e ter smartphone. Excluíram-se do estudo os estudantes considerados inaptos a responder aos questionários por incapacidade física, mental ou psicológica. Os alunos que não aceitaram participar do estudo foram considerados como casos ausentes, para, posterior, análise de sensibilidade.

Considerado uma referência no meio acadêmico, o curso de Medicina da Unichristus existe há 14 anos e tem uma grade curricular híbrida (aulas convencionais e problem based learning). Os alunos foram abordados pessoalmente no período de março a junho de 2019. Após concordarem em participar do estudo, assinaram o Termo de Consentimento Livre e Esclarecido e foram submetidos ao preenchimento de um questionário sociodemográfico e dois questionários validados: Depression Anxiety and Stress Scale 21 (DASS-21) e Nomophobia Questionnaire (NMP-Q).

\section{Medidas}

\section{NMP-Q e DASS-21}

A nomofobia foi mensurada por meio do NMP-Q ${ }^{6}$. Este instrumento tem 20 questões, todas baseadas na escala do tipo Likert de 7 pontos: de 1 (discordo totalmente) a 7 (concordo plenamente). Essa escala foi validada para o português brasileiro em $2020^{15}$. A interpretação, segundo os autores, é baseada na quantidade de escores marcados. A pontuação total no NMP-Q é de 20 no mínimo $(20 * 1)$ ou $140(7 * 20)$ no máximo, e os resultados podem ser classificados assim: menos de 20 pontos: ausência de nomofobia; 21-59: nível leve de nomofobia; 60-99: nível moderado de nomofobia; e 100-140: nomofobia grave.

A depressão, a ansiedade e o estresse foram mensurados pela DASS-2 $1^{16}$, uma versão simplificada da DASS. O questionário DASS-21 foi validado para o português brasileiro ${ }^{17}$. O questionário é composto de um conjunto de três subescalas, do tipo Likert de 4 pontos - de 0 (não se aplicou de 
maneira alguma) a 3 (aplicou-se muito ou na maioria do tempo) - de autorresposta, em que os sintomas de estresse, depressão e ansiedade se agrupam. A primeira subescala é definida pela presença de afetos negativos, como humor deprimido, insônia, desconforto e irritabilidade, que são sintomas inespecíficos associados com estresse; a segunda engloba fatores que constituem estruturas que representam sintomas específicos para depressão (anedonia, ausência de afeto positivo); por fim, a última estrutura refere-se aos sintomas específicos de ansiedade (tensão somática e hiperatividade).

\section{Índice de Rendimento Acadêmico (IRA)}

O IRA é o fruto de uma complexa operação matemática que resulta em uma nota média do aluno no semestre e funciona como um índice de referência para acompanhamento pedagógico na Unichristus. Trata-se de uma nota obtida por meio de um cálculo, representando uma nota média do aluno no curso até o dado momento. O IRA é calculado com a seguinte fórmula:

$$
\begin{gathered}
A P I=\frac{[F G(D 1 S 1)+F G(D 2 S 1)+\cdots+F G(D N S 1)]}{N D S 1} \\
+\frac{[F G(D 1 S 2)+F G(D 2 S 2)+\cdots+F G(D N S 2)]}{N D S 2} \ldots \\
+\frac{[F G(D 1 S n)+F G(D 2 S n)+\cdots+F G(D N S n)]}{N D S n}
\end{gathered}
$$

Nessa fórmula:

- A sigla NF(D1S1) significa a nota final (ou média final) da primeira disciplina (módulo) obtida no histórico do primeiro semestre do aluno somente para as disciplinas APROVADAS no histórico, exceto a disciplina denominada Atividade Complementar; NF (D2S1) é a nota final (ou média final) da segunda disciplina (módulo) obtida no histórico do primeiro semestre do aluno somente para as disciplinas APROVADAS no histórico, exceto a disciplina denominada Atividade Complementar. E assim sucessivamente até a última disciplina aprovada pelo aluno no primeiro semestre que será NF(DNS1), que significa a nota final (ou média final) da $\mathrm{N}^{a}$ (enésima) disciplina (módulo) obtida no histórico do primeiro semestre do aluno somente para as disciplinas aprovadas no histórico, exceto a disciplina denominada Atividade Complementar.

- A sigla QDS1 significa a quantidade de disciplinas (somente aprovadas e reprovadas, exceto as disciplinas denominadas "Atividade Complementar") cursadas no primeiro semestre do histórico do aluno. A sigla NF(D1S2) significa a nota final (ou média final) da primeira disciplina (módulo) obtida no histórico do segundo semestre do aluno somente para as disciplinas APROVADAS no histórico, exceto a disciplina denominada Atividade Complementar.

- A sigla NF(D2S2) significa a nota final (ou média final) da segunda disciplina (módulo) obtida no histórico do segundo semestre do aluno somente para as disciplinas aprovadas no histórico, exceto a disciplina denominada Atividade Complementar. E assim sucessivamente até a última disciplina aprovada pelo aluno no segundo semestre que será NF(DNS2), que significa nota final (ou média final) da $\mathrm{N}^{\text {a }}$ disciplina/módulo obtida no histórico do segundo semestre do aluno somente para as disciplinas aprovadas no histórico, exceto a disciplina denominada Atividade Complementar. A sigla QDS2 significa a quantidade de disciplinas (somente aprovadas e reprovadas, exceto as disciplinas denominadas "Atividade Complementar") cursadas no segundo semestre do histórico do aluno.

- A sigla NF(D1SN) significa a nota final (ou média final) da primeira disciplina (módulo) obtida no histórico do $\mathrm{N}^{\circ}$ semestre do aluno somente para as disciplinas aprovadas no histórico, exceto a disciplina denominada Atividade Complementar. A NF(D2SN) é a nota final da segunda disciplina/módulo obtida no histórico do $\mathrm{N}^{\circ}$ semestre do aluno somente para as disciplinas aprovadas no histórico, exceto a disciplina denominada Atividade Complementar. $\mathrm{E}$ assim sucessivamente até a última disciplina aprovada pelo aluno do $\mathrm{N}^{\circ}$ semestre que será NF(DNSN) que significa a nota final da $\mathrm{N}^{\mathrm{a}}$ disciplina/ módulo obtida no histórico do $\mathrm{N}^{\circ}$ semestre do aluno somente para as disciplinas aprovadas no histórico, exceto a disciplina denominada Atividade Complementar. A sigla QDSN significa a quantidade de disciplinas (somente aprovadas e reprovadas, exceto as disciplinas denominadas "Atividade Complementar") cursadas no $\mathrm{N}^{\circ}$ semestre do histórico do aluno. A sigla QSL significa a quantidade de semestres letivos cursados pelo aluno.

\section{Variáveis sociodemográficas}

O questionário sociodemográfico foi composto por cinco questões que abordavam o semestre em que o aluno estaria cursando no momento, o grupo de tutoria (um ambiente em que o aprendizado é baseado no problem based learning, uma metodologia ativa de busca do conhecimento) e o número do aluno, que foi necessário para que pudéssemos interligar os dados do IRA de modo completamente anônimo. Além desses, foram colhidos idade e sexo do aluno. 


\section{Análise estatística}

Os escores de nomofobia, ansiedade, estresse e depressão foram expressos em forma de média e desvio padrão e comparados entre as categorias de IRA por meio do teste de Mann-Whitney, bem como categorizados conforme orientado em seus artigos de validação. O IRA dos alunos foi dividido em tercis $(<7,8 ; 7,8-8,3 ;>8,3)$ para expressão das frequências absoluta e percentual e cruzamento com os fatores sociodemográficos por meio do teste qui-quadrado de Pearson ou teste exato de Fisher para categóricas e Mann-Whitney para numéricas. Realizaramse também análises de correlação com extrapolação cúbica. Fez-se análise de sensibilidade com os casos que recusaram a participação (13 alunos), e a variação nas associações não foi significativa. Consideraram-se significativos valores de $p$ inferiores a 0,05. Os dados obtidos na coleta foram tabulados e analisados pelo software IBM SPSS Statistics for Windows, version 23.0 (Armonk, NY: IBM Corp. IBM Corp. Released 2015).

\section{Aspectos éticos}

Este estudo foi aprovado pelo Comitê de Ética em Pesquisa da Unichristus, sendo protocolado com o Certificado de Apresentação para Apreciação Ética (CAAE) no 87329618.4.0000.5049. Todas as informações foram coletadas com base na Resolução do Conselho Nacional de Saúde (CNS) no 466/2012 que normatiza os princípios de ética em pesquisa com seres humanos. Todos os participantes receberam e assinaram o Termo de Consentimento Livre e Esclarecido.

\section{RESULTADOS}

Neste estudo, obteve-se uma amostra de 292 estudantes de Medicina da Unichristus, dos quais 59,9\% eram do sexo feminino e $40,1 \%$ do sexo masculino, e $32,9 \%$ faziam parte do primeiro ao terceiro semestre, e $67,1 \%$, do quarto ao oitavo semestre. Ademais, 69,6\% tinham idade acima dos 20 anos (média 22, desvio padrão 4).

Praticamente todos os alunos $(99,7 \%)$ apresentaram algum grau de nomofobia: $64,5 \%$ com nível moderado ou grave e $11,8 \%$ com nível grave. Mais de $50 \%$ dos estudantes apresentaram graus maiores do nível leve (de mínimo a muito grave) de estresse; e 19,5\% e 11,2\%, ansiedade e depressão graves ou muito graves, respectivamente (Tabela 1).

Quando se analisa o perfil de utilização de celular pelos estudantes, observa-se que a maioria deles $(60,5 \%)$ o usa, no total acumulado, durante um tempo menor que quatro horas e que isso se associa com o IRA, com o menor uso associado ao melhor IRA ( $p=0,021)$. Entretanto, a maioria checa o celular a cada 5, 10, ou 20 minutos. Entre as finalidades, as mais prevalentes foram conversar ou enviar mensagens para família e amigos, seguido por pesquisar informações, com mais de $80 \%$ dos entrevistados utilizando para esse fim. O uso de e-mail, prevalente em quase $70 \%$ dos alunos, foi associado ao pior IRA (valor de $\mathrm{p}$ de 0,04 ), bem como o uso para agendar reuniões $(p=0,001)$ (Tabela 2 ).

Quando se analisa a correlação dos escores no NMP-Q com os escores da DASS-21, observa-se que aumentos nessa pontuação leva à elevação do escore geral da DASS $(p<0,001)$ (Gráfico 1), bem como em cada um dos domínios de depressão $(p<0,001)$, ansiedade $(p<0,001)$ e estresse $(p<0,001)$.

Tabela 1. Prevalência de nomofobia, estresse, ansiedade e depressão entre os 292 estudantes avaliados.

\begin{tabular}{lc}
\hline Escore NMQ & $\begin{array}{c}\mathbf{n}(\%) \text { ou média } \pm \\
\text { desvio padrão }\end{array}$ \\
\hline NMQ em categorias & $71,2 \pm 24,1$ \\
Sem nomofobia & $1(0,3)$ \\
Nomofobia leve & $104(35,1)$ \\
Nomofobia moderada & $156(52,7)$ \\
Nomofobia grave & $35(11,8)$ \\
\hline IRA em tercis & $112(38,4)$ \\
1 & $92(31,5)$ \\
2 & $88(30,1)$ \\
3 & \\
\hline DASS-21 & $16,4 \pm 10,8$ \\
Escore domínio estresse & $8,2 \pm 9,2$ \\
Escore domínio ansiedade & $8,6 \pm 9,1$ \\
Escore domínio depressão
\end{tabular}

Domínio estresse em categorias

Normal/leve

$131(47,2)$

Mínimo

$37(13,3)$

Moderado

$48(17,3)$

Grave

$40(14,4)$

Muito grave

$21(7,5)$

\begin{tabular}{lc}
\hline Domínio ansiedade em categorias & \\
Normal/leve & $154(55,7)$ \\
Mínimo & $23(8,3)$ \\
Moderado & $45(16,3)$ \\
Grave & $23(8,3)$ \\
Muito grave & $31(11,2)$
\end{tabular}

Domínio depressão em categorias

Normal/leve

$178(64,4)$

Mínimo

$35(12,6)$

Moderado

$32(11,5)$

Grave

$13(4,7)$

Muito grave

$18(6,5)$ 
Por fim, quando se analisa a associação entre a DASS21, em seus domínios, e o IRA, verifica-se que o primeiro tercil de IRA, que representa os alunos com menor rendimento, apresenta maiores escores e piores resultados da DASS-21 em todos os domínios avaliados. A média do escore de ansiedade em alunos no primeiro tercil é de 9,9, contra 6,3 nos alunos do tercil superior (valor de $\mathrm{p}$ de 0,044 ), e a prevalência de estresse grave ou muito grave é de $31,6 \%$ em alunos do primeiro tercil, sendo cerca do dobro do encontrado nos alunos de terceiro tercil (16,2\%), valor de p de 0,035 (Tabela 3).

Tabela 2. Perfil de utilização do celular e associação do uso com o IRA.

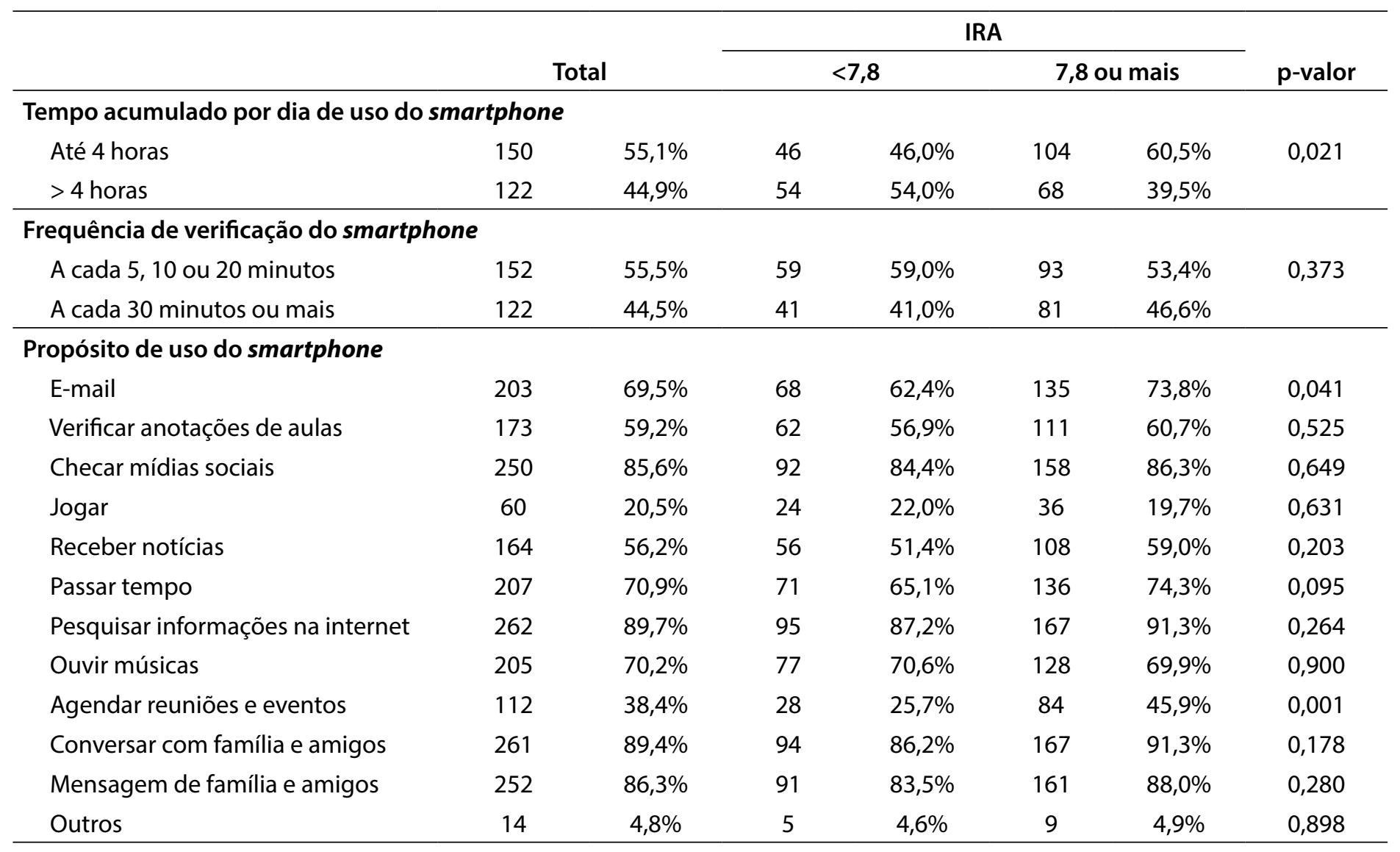

Gráfico 1. Dispersão simples de DASS-21 por NMQ.

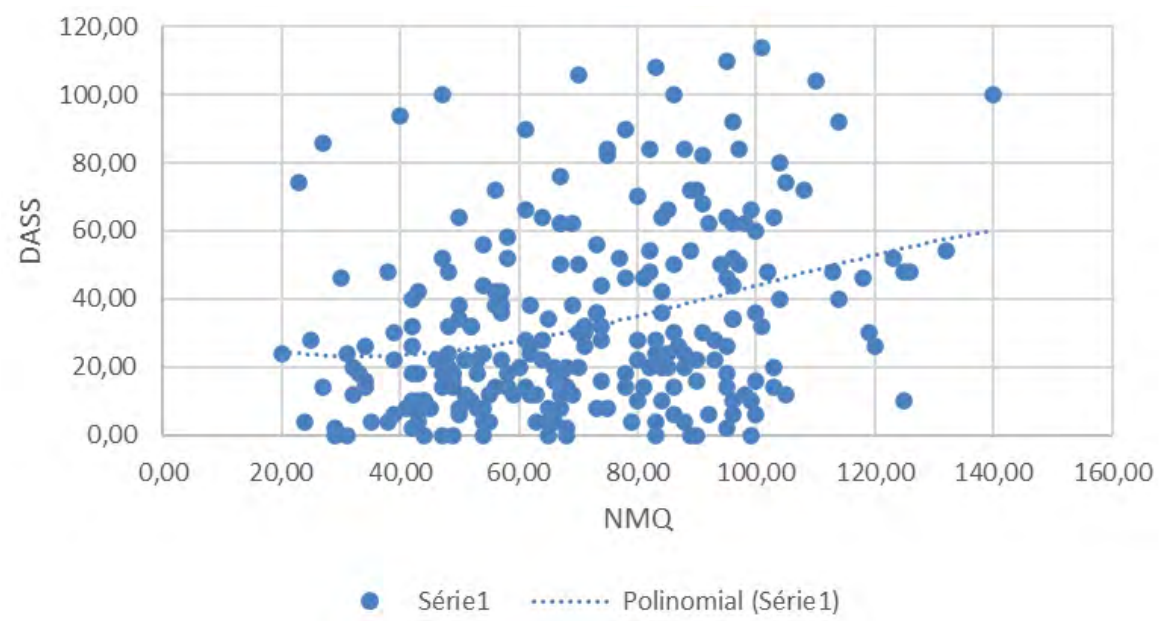


Tabela 3. Associação das medidas nos domínios avaliados da DASS-21 e os tercis do IRA.

\begin{tabular}{|c|c|c|c|c|}
\hline & \multicolumn{3}{|c|}{ IRA em tercis } & \multirow{3}{*}{ Valor de $p$} \\
\hline & Primeiro & Segundo & Terceiro & \\
\hline & $\begin{array}{c}\mathrm{n}(\%) \text { ou média } \pm \\
\text { desvio padrão }\end{array}$ & $\begin{array}{l}\mathrm{n}(\%) \text { ou média } \pm \\
\text { desvio padrão }\end{array}$ & $\begin{array}{c}\mathrm{n}(\%) \text { ou média } \pm \\
\text { desvio padrão }\end{array}$ & \\
\hline Escore domínio estresse & $18,1 \pm 12,35$ & $16 \pm 9,98$ & $14,7 \pm 9,33$ & 0,223 \\
\hline Escore domínio ansiedade & $9,9 \pm 10,33$ & $8 \pm 8,27$ & $6,3 \pm 7,91$ & 0,044 \\
\hline Escore domínio depressão & $10,1 \pm 9,87$ & $9,3 \pm 9,94$ & $6,3 \pm 6,6$ & 0,059 \\
\hline Domínio estresse em categorias & & & & 0,035 \\
\hline Normal/leve & $41(40,5)$ & $42(50)$ & $45(52,3)$ & \\
\hline Mínimo & $13(12,8)$ & $11(13)$ & $12(13,9)$ & \\
\hline Moderado & $15(14,8)$ & $17(20,2)$ & $15(17,4)$ & \\
\hline Grave & $19(18,8)$ & $7(8,3)$ & $14(16,2)$ & \\
\hline Muito grave & $13(12,8)$ & $7(8,3)$ & $0(0)$ & \\
\hline Domínio ansiedade em categorias & & & & 0,326 \\
\hline Normal/leve & $49(49)$ & $45(53,5)$ & $56(65,1)$ & \\
\hline Mínimo & $6(6)$ & $9(10,7)$ & $8(9,3)$ & \\
\hline Moderado & $19(19)$ & $14(16,6)$ & $11(12,7)$ & \\
\hline Grave & $11(11)$ & $8(9,5)$ & $4(4,6)$ & \\
\hline Muito grave & $15(15)$ & $8(9,5)$ & $7(8,1)$ & \\
\hline Domínio depressão em categorias & & & & 0,203 \\
\hline Normal/leve & $56(56)$ & $53(62,3)$ & $64(75,2)$ & \\
\hline Mínimo & $16(16)$ & $10(11,7)$ & $9(10,5)$ & \\
\hline Moderado & $12(12)$ & $11(12,9)$ & $9(10,5)$ & \\
\hline Grave & $7(7)$ & $5(5,8)$ & $1(1,1)$ & \\
\hline Muito grave & $9(9)$ & $6(7)$ & $2(2,3)$ & \\
\hline
\end{tabular}

\section{DISCUSSÃO}

O presente estudo analisou a relação de nomofobia com depressão, ansiedade, estresse e IRA. Avaliaram-se 292 estudantes do primeiro ao oitavo semestre do curso de Medicina da Unichristus. Nossa amostra foi composta de alunos de uma ampla faixa etária e dos gêneros masculinos e femininos. Verificou-se associação importante de nomofobia com níveis de ansiedade, depressão e estresse, e os três últimos foram associados com pior rendimento acadêmico. Além disso, constatou-se que os usos de celular mais associados com pior rendimento foram checar e-mail e marcar reuniões.

Em nosso estudo, evidenciou-se que os estudantes do curso de Medicina tinham uma correlação positiva entre nomofobia e depressão, ou seja, quanto maiores os escores de nomofobia, maiores os de depressão. Corroborando os nosso achados, um estudo realizado com 329 estudantes norteamericanos demonstrou que o uso problemático de smartphone tem uma relação com a severidade da depressão ${ }^{18}$. Quanto à correlação encontrada no presente estudo, uma pesquisa feita com estudantes do curso de Enfermagem da Índia evidenciou também que a nomofobia aumenta os níveis de depressão devido à pressão que o indivíduo sofre por ter que estar continuamente conectado ${ }^{19}$. Ademais, ainda reforçando os nossos achados, uma revisão sistemática e metanálise chinesa encontrou, em um total de 13 estudos, uma correlação entre uso problemático de smartphone e depressão ${ }^{20}$. Tais evidências sugerem que provavelmente exista uma correlação entre o uso aumentado de smartphone e a depressão.

Além desses achados, um estudo mostrou que estudantes com uso de smartphone prolongado (mais de quatro horas) têm um menor rendimento acadêmico. Além disso, constatou-se que os estudantes que usam o smartphone para olhar e-mails e agendar reuniões e eventos têm um rendimento acadêmico maior, com o IRA > 7,8 em média. Tal fato pode acontecer devido a um uso mais responsável do smartphone, pois, provavelmente, usá-lo para agendar reuniões e ver e-mail representa consultas pontuais ao celular, ou seja, não há feedback, de modo que o aluno fica menos tempo conectado. O papel do smartphone no rendimento acadêmico é alvo de diversos estudos na atualidade. Em uma pesquisa de larga escala 
realizada em uma universidade pública chinesa, constatouse uma correlação entre o uso exacerbado de smartphone e a performance acadêmica negativa dos estudantes, via nomofobia ${ }^{21}$. Por sua vez, uma pesquisa que utilizou o mesmo instrumento de pesquisa deste estudo (NMP-Q), com uma amostra de 157 estudantes, não encontrou a associação entre uso de smartphone e um pior rendimento acadêmico entre alunos de um curso de Fisioterapia 5 . No presente estudo, não se encontrou uma correlação direta entre nomofobia e IRA, porém evidenciou-se uma correlação indireta, visto que os alunos que apresentam o IRA baixo têm relação com ansiedade, que, por sua vez, correlaciona-se estatisticamente com nomofobia.

Como demonstrado neste estudo, constatou-se uma associação estatisticamente significativa entre nomofobia e ansiedade, evidenciando que alunos com altos índices de nomofobia tendem a ter maiores níveis de ansiedade. Essa relação foi reforçada em uma revisão sistemática com 42 artigos, na qual se utilizou o mesmo instrumento do presente estudo para mensuração (NMP-Q). Na grande maioria dos artigos estudados, encontrou-se uma correlação positiva entre nomofobia e ansiedade ${ }^{22}$. O uso exacerbado de smartphone e sua relação com a nomofobia, como visto em nosso artigo, são temas estudados em todo o mundo. Em uma análise realizada na China com adolescentes, foi proposta a existência de uma importante associação entre ansiedade social e dependência de smartphone ${ }^{23}$. Confirmando os dados encontrados em nosso artigo, o uso de celulares tem sido descrito com altos níveis de ansiedade, sendo o tempo de uso o principal fator associado ${ }^{24}$.

Além da relação entre nomofobia e ansiedade, no presente estudo, os níveis de estresse dos indivíduos também foram correlacionados com a nomofobia, ou seja, quanto maior um, maior foi o nível do outro. Tal associação implica acreditar que o uso exacerbado de smartphone leva a mecanismos de estresse, tendo um impacto significativo na qualidade de vida e na saúde mental do indivíduo. Uma revisão sistemática e metanálise realizada com 41 artigos demonstrou que o uso problemático de smartphone aumenta os níveis de estresse percebido entre estudantes ${ }^{22}$. Ademais, uma pesquisa com 270 usuários de smartphone evidenciou que nomofobia e estresse têm uma associação intrínseca, pois se observou uma correlação entre nomofobia, estresse e condições de trabalho ${ }^{8}$.

Alguns achados deste estudo mostram que depressão, ansiedade e estresse estão correlacionados entre si: quanto maior for o nível de uma condição, maior será o nível das outras. Corroborando os nossos achados, uma investigação avaliou a depressão, a ansiedade e o estresse entre estudantes de Medicina na Índia e encontrou uma alta correlação entre essas três condições na amostra, visto que essas doenças têm, provavelmente, uma correlação fisiopatológica entre si. Em contrapartida, uma pesquisa com estudantes de Medicina realizada na Nigéria não constatou correlação entre depressão, ansiedade e estresse $\mathrm{e}^{25}$.

Este estudo tem algumas limitações. Provavelmente a mais importante é o uso da metodologia transversal, que pode levar à causalidade reversa. Acreditamos que esse não seja o caso do presente estudo por termos avaliado estudantes desde o primeiro semestre (o grupo mais representativo), e assim o impacto do IRA na geração de estresse, ansidade, depressão ou nomofobia ainda não é temporalmente provável. Tivemos uma taxa de valores ausentes aceitável e utilizamos análises de sensibilidade que demonstraram pouco efeito nas associações. Como foi um questionário autoaplicado, poderíamos experimentar o viés de informação, mas, durante a aplicação, a informação de sigilo foi garantida. Por fim, ainda que o IRA não seja uma medida perfeita de desempenho acadêmico, ele consegue registrar de forma aceitável e estável o rendimento dos estudantes.

\section{CONCLUSÕES}

Nosso estudo sugere que a nomofobia pode provavelmente aumentar a ansiedade e, consequentemente, levar a uma baixa do rendimento acadêmico. Diante disso, políticas de tecnologias educacionais são de suma importância dentro do ambiente acadêmico, tendo como objetivos alertar os alunos sobre o uso abusivo do celular, conduzir uma adicção e auxiliar o discente a procurar algum plano terapêutico. Além disso, nosso estudo constatou que a nomofobia tem correlação com ansiedade, depressão e estresse. As instituições de ensino precisam alertar alunos e ajudá-los no tocante a esse problema, visto que tais comorbidades podem trazer consequências extremamente danosas ao indivíduo, como o suicídio. Ademais, outras pesquisas serão necessárias para a confirmação dos achados obtidos neste estudo, de modo a lidar de uma maneira mais efetiva com esse assunto.

\section{CONTRIBUIÇÃO DOS AUTORES}

Marcos Kubrusly e Hermano Alexandre Lima Rocha conceberam e escreveram o artigo. Paulo Goberlânio de Barros Silva, Gabriel Vidal de Vasconcelos, Emanuel Delano Lima Gonçalves Leite e Priscilla de Almeida Santos participaram da coleta e análise de dados. Hermano Alexandre Lima Rocha realizou as análises estatísticas. Hermano Alexandre Lima Rocha, Marcos Kubrusly, Paulo Goberlânio de Barros Silva, Gabriel Vidal de Vasconcelos e Emanuel Delano Lima Gonçalves Leite leram, revisaram e aprovaram a versão final do manuscrito.

\section{CONFLITO DE INTERESSES}

Declaramos não haver conflito de interesses. 


\section{FINANCIAMENTO}

\section{Declaramos não haver financiamento.}

\section{REFERÊNCIAS}

1. Poushter J. Smartphone ownership and internet usage continues to climb in emerging economies. Pew Research Center; 2016 [access in 25 jun 2021]. Available from: https://www.pewresearch.org/global/2016/02/22/ smartphone-ownership-and-internet-usage-continues-to-climb-inemerging-economies.

2. Wolniewicz CA, Tiamiyu MF, Weeks JW, Elhai JD. Problematic smartphone use and relations with negative affect, fear of missing out, and fear of negative and positive evaluation. Psychiatry Res. 2018;262:618-23.

3. Melumad S, Pham MT. The smartphone as a pacifying technology. J Consum Res. 2020;47(2):237-255.

4. Alhassan AA, Alqadhib EM, Taha NW, Alahmari RA, Salam M, Almutairi AF. The relationship between addiction to smartphone usage and depression among adults: a cross sectional study. BMC Psychiatry. 2018;18(1):148.

5. Ahmed S, Pokhrel N, Roy S, Samuel AJ. Impact of nomophobia: a nondrug addiction among students of physiotherapy course using an online crosssectional survey. Indian J Psychiatry. 2019;61(1):77-80.

6. Yildirim C, Correia A-P. Exploring the dimensions of nomophobia: development and validation of a self-reported questionnaire. Comput Human Behav. 2015;49:130-7.

7. King A, Guedes E, Pedro Neto J, Guimarães F, Nardi A. Nomophobia: clinical and demographic profile of social network excessive users. J Addict Res Ther. 2017;8:339.

8. Tams S, Legoux R, Léger P-M. Smartphone withdrawal creates stress: a moderated mediation model of nomophobia, social threat, and phone withdrawal context. Comput Human Behav. 2018;81:1-9.

9. Durak HY. Investigation of nomophobia and smartphone addiction predictors among adolescents in Turkey: demographic variables and academic performance. Soc Sci J. 2019;56(4):492-517.

10. Farooqui IA, Pore $P$, Gothankar J. Nomophobia: an emerging issue in medical institutions? J Ment Health. 2018;27(5):438-41.

11. Chethana K, Nelliyanil M, Anil M. Prevalence of nomophobia and its association with loneliness, self happiness and self esteem among undergraduate medical students of a medical college in Coastal Karnataka. Indian J Public Health Res Dev. 2020;11(3):523-9.

12. Chun JW, Choi J, Cho H, Choi MR, Ahn KJ, Choi JS, et al. Role of Frontostriatal Connectivity in Adolescents With Excessive Smartphone Use. Front Psychiatry. 2018;9:437.
13. Horvath J, Mundinger C, Schmitgen MM, Wolf ND, Sambataro F, Hirjak D, et al. Structural and functional correlates of smartphone addiction. Addict Behav. 2020;105:106334.

14. Prasad M, Patthi B, Singla A, Gupta R, Saha S, Kumar JK, et al. Nomophobia: a cross-sectional study to assess mobile phone usage among dental students. J Clin Diagn Res. 2017;11(2):ZC34-ZC9.

15. Rocha HAL, de Almeida Santos P, de Vasconcelos GV, Leite EDLG, Kubrusly M. Adaptação transcultural e validação do Nomophobia Questionnaire (NMP-Q) para a língua portuguesa (NMP-Q-BR). J Health Biol Sci (Online). 2020;8(1):1-6.

16. Lovibond PF, Lovibond $\mathrm{SH}$. The structure of negative emotional states: comparison of the Depression Anxiety Stress Scales (DASS) with the Beck Depression and Anxiety Inventories. Behav Res Ther. 1995;33(3):335-43.

17. Vignola RC, Tucci AM. Adaptation and validation of the Depression, Anxiety and Stress Scale (DASS) to Brazilian Portuguese. J Affect Disord. 2014;155:104-9.

18. Elhai JD, Gallinari EF, Rozgonjuk D, Yang H. Depression, anxiety and fear of missing out as correlates of social, non-social and problematic smartphone use. Addict Behav. 2020;105:106335.

19. Sagar K. Smartphone addiction: nomophobia. Asian Journal of Nursing Education and Research. 2019;9(4):583-7.

20. Yang J, Fu X, Liao X, Li Y. Association of problematic smartphone use with poor sleep quality, depression, and anxiety: a systematic review and metaanalysis. Psychiatry Res. 2020;284:112686.

21. Fu S, Chen $X$, Zheng $H$. Exploring an adverse impact of smartphone overuse on academic performance via health issues: a stimulus-organismresponse perspective. Behav Inf Technol. 2020;40(7):663-75.

22. Rodríguez-García AM, Moreno-Guerrero AJ, López Belmonte J. Nomophobia: an Individual's growing fear of being without a smartphone: a systematic literature review. Int J Environ Res Public Health. 2020;17(2):580.

23. Kong F, Qin J, Huang B, Zhang H, Lei L. The effect of social anxiety on mobile phone dependence among Chinese adolescents: a moderated mediation model. Child Youth Serv Rev. 2020;108:104517.

24. Darvishi M, Noori M, Nazer MR, Sheikholeslami S, Karimi E. Investigating different dimensions of nomophobia among medical students: a crosssectional study. Open Access Maced J Med Sci. 2019;7(4):573-8.

25. Coker AO, Coker OO, Sanni D. Sociodemographic correlates and symptoms of depression, anxiety and stress among a sample of nigerian medical students. Nigerian Journal of Basic and Clinical Sciences. 2018;15(1):58. 